\title{
ACOUSTIC BOUNDARY CONDITIONS
}

\author{
BY J. THOMAS BEALE AND STEVEN I. ROSENCRANS
}

Communicated by H. Weinberger, May 28, 1974

In this paper we announce results in the study of the wave equation

$$
\phi_{t t}=\Delta \phi
$$

subject to what we call acoustic boundary conditions. The physical model giving rise to these conditions is that of a gas undergoing small irrotational perturbations from rest in a domain $G$ with smooth compact boundary. We assume that each point of the surface $\partial G$ acts like a spring in response to the excess pressure in the gas, and that there is no transverse tension between neighboring points of $\partial G$, i.e., the "springs" are independent of each other. (Such a surface is called locally reacting; see [2, pp. 259-264] .)

If the boundary has mass per unit area $m$, resistivity $d$, and spring constant $k$ (all nonnegative functions on $\partial G$ ), then the displacement $\delta(x, t)$ into the domain of a point $x \in \partial G$ at time $t$ must satisfy the spring equation

$$
m \delta_{t t}+d \delta_{t}+k \delta=- \text { excess pressure }=\rho_{0} \phi_{t},
$$

where $\rho_{0}$ is the unperturbed density of the gas and $\phi(x, t)$ is the velocity potential. Continuity of the normal velocity between the gas and the boundary implies the relation $\delta_{t}(x, t)=-\phi_{n}(t, x-n \delta(x, t)), x \in \partial G$, where $n$ is the outward normal. We consider here the linearized approximation obtained by assuming $\delta$ is small (this is consistent with the linearization leading to the wave equation). Thus we assume

$$
\delta_{t}(x, t)=-\phi_{n}(x, t) .
$$

Note that if $d$ and $k$ are zero, (2) and (3) imply $m \phi_{n t}+\rho_{0} \phi_{t}=0$; thus the excess pressure satisfies the Robin boundary condition.

If $\phi$ and $\delta$ are smooth solutions of (1)-(3), and $\phi$ has compact support in space for each $t$ if $G$ is unbounded, it is easy to see that the energy form

AMS (MOS) subject classifications (1970). Primary 35L05, 35P25, $76 \mathrm{Q} 05$. 


$$
E(t)=\int_{G}\left(\rho_{0}|\nabla \phi|^{2}+\frac{\rho_{0}}{c^{2}} \phi_{t}^{2}\right)+\int_{\partial G}\left(m \phi_{n}^{2}+k \delta^{2}\right)
$$

is nonincreasing in $t$, and if $d$ is zero it is constant. The four terms represent the kinetic and potential energies of the acoustic wave and the displacement of the boundary. We are thus led to consider solutions as four-vectors $\left(\phi, \phi_{t}, \phi_{n}, \delta\right)$ in the Hilbert space $H$ which is the closure of $C_{0}^{\infty}(\bar{G})+$ $C_{0}^{\infty}(\bar{G})+C^{\infty}(\partial G)+C^{\infty}(\partial G)$ in the norm corresponding to (4). An operator $A$ can be defined on $H$ so that, for smooth $\phi$ and $\delta$, (1)-(3) are equivalent to $u(t) \in D(A)$ and $u_{t}=A u$, where $u=\left(\phi, \phi_{t}, \phi_{n}, \delta\right) . A$ is closed, densely defined, and maximal dissipative; therefore by a theorem of Lumer and Phillips it generates a contraction semigroup which provides a solution of the initial value problem.

For a bounded domain it might be expected, by analogy with the usual boundary conditions for the wave equation, that $A$ has discrete spectrum consisting of eigenvalues; however, this is not so. The following results describe the spectrum of $A$ in the case where $m, k$, and $d$ are constant on $\partial G$ and $k>0, m>0 . \omega_{1}$ and $\omega_{2}$ are the roots of $m \omega^{2}+d \omega+k$.

TheOREM 1. $\left(m A^{2}+d A+k\right)(1-A)^{-3}$ is compact. As a consequence, the resolvent of $A$ is meromorphic on $\mathbf{C}-\left\{\omega_{1}, \omega_{2}\right\}$, and its poles are eigenvalues of $A$.

THEOREM 2. $\omega_{1}$ and $\omega_{2}$ are essential singularities of the resolvent of $A$.

THEOREM 3. If $d=0$, there exists a sequence of eigenvalues of $A$ converging to $\omega_{1}\left(\right.$ or $\left.\omega_{2}\right)$.

If $\partial G$ is a sphere, the conclusion of Theorem 3 holds even if $d>0$. Whether it holds for arbitrary boundary with $d>0$ is not known. For exterior domains there are results similar to Theorems 1 and 2 for the "outgoing" resolvent (see [1]).

Finally we have considered scattering theory for this model in an exterior domain with the wave equation in free space as the unperturbed system. If $m, k$, and $d$ are variable but $d$ is bounded below by a positive constant, the methods of Lax and Phillips can be applied to obtain an energy decay result like Theorem 9.5 of [1]. It follows from the abstract theory that wave operators and a scattering operator exist. We expect that in the case $d=0$ the existence of these operators can be shown by a different method. 


\section{REFERENCES}

1. P. D. Lax and R. S. Phillips, Scattering theory for dissipative hyperbolic systems, J. Functional Analysis 14 (1973), 172-235.

2. P. M. Morse and K. U. Ingard, Theoretical acoustics, McGraw-Hill, New York, 1968.

DEPARTMENT OF MATHEMATICS, TULANE UNIVERSITY, NEW ORLEANS, LOUISIANA 70118 